\title{
Single-shot BOTDA based on an optical chirp chain probe wave for distributed ultrafast measurement
}

\author{
Dengwang Zhou (1)', Yongkang Dong $\mathbb{D}^{1}$, Benzhang Wang ${ }^{1}$, Chao Pang ${ }^{1}$, Dexin Ba', Hongying Zhang ${ }^{2}$, Zhiwei Lu',
} Hui $\mathrm{Li}^{3}$ and Xiaoyi Bao (i) ${ }^{4}$

\begin{abstract}
Brillouin optical time-domain analysis (BOTDA) requires frequency mapping of the Brillouin spectrum to obtain environmental information (e.g., temperature or strain) over the length of the sensing fiber, with the finite frequencysweeping time-limiting applications to only static or slowly varying strain or temperature environments. To solve this problem, we propose the use of an optical chirp chain probe wave to remove the requirement of frequency sweeping for the Brillouin spectrum, which enables distributed ultrafast strain measurement with a single pump pulse. The optical chirp chain is generated using a frequency-agile technique via a fast-frequency-changing microwave, which covers a larger frequency range around the Stokes frequency relative to the pump wave, so that a distributed Brillouin gain spectrum along the fiber is realized. Dynamic strain measurements for periodic mechanical vibration, mechanical shock, and a switch event are demonstrated at sampling rates of $25 \mathrm{kHz}, 2.5 \mathrm{MHz}$ and $6.25 \mathrm{MHz}$, respectively. To the best of our knowledge, this is the first demonstration of distributed Brillouin strain sensing with a wide-dynamic range at a sampling rate of up to the $\mathrm{MHz}$ level.
\end{abstract}

\section{Introduction}

In modern industry ${ }^{1-3}$, geophysical research ${ }^{4-6}$, the health monitoring of civil infrastructures ${ }^{1,2}$, and the motion capturing of robot hands ${ }^{7,8}$ and the human body ${ }^{9}$, a truly distributed ultrafast measurement, are widely required for real-time monitoring of distributed strain or temperature information. Compared with conventional electric sensing networks, optical fiber sensing offers an attractive solution due to several advantages: smallness in size, low cost, long sensing range, chemical inertness, and immunity to external-electromagnetic interference ${ }^{10}$, where the optical fiber simultaneously acts as the optical transmitting medium and millions of sensing points.

Correspondence: Yongkang Dong (aldendong@gmail.com)

${ }^{1}$ National Key Laboratory of Science and Technology on Tunable Laser, Harbin Institute of Technology, 150001 Harbin, China

${ }^{2}$ Department of Optoelectronic Information, Science and Engineering, Harbin University of Science and Technology, 150080 Harbin, China

Full list of author information is available at the end of the article.
Brillouin-based distributed sensors have been widely reported since the $1990 \mathrm{~s}^{2,11,12}$. Generally, two counterpropagating optical waves (i.e., pump wave and probe wave) are launched into a fiber under test (FUT). As the frequency difference between these two waves approaches the local Brillouin frequency shift (BFS) of the FUT, the optical power transferred from the high-frequency light wave to the low-frequency light wave reaches a maximum. As a result, the Brillouin gain spectrum (BGS) can be obtained by sweeping the detuned frequency. Then, the BFS, which is dependent on both applied strain and temperature along the FUT $2,10,11,13-15$, can be calculated by curve fitting the BGS. The process of frequency tuning to obtain the BGS is time-consuming. Distributed fast strain measurement has been previously realized using several techniques: Brillouin optical correlation-domain analysis $(\mathrm{BOCDA})^{3,16-18}$, Brillouin optical correlation-domain reflectometry $(\mathrm{BOCDR})^{3,19-25}$, and Brillouin optical timedomain analysis (BOTDA) $)^{12,26-32}$.

\section{(c) The Author(s) 2018}

(c) (i) Open Access This article is licensed under a Creative Commons Attribution 4.0 International License, which permits use, sharing, adaptation, distribution and reproduction cc) in any medium or format, as long as you give appropriate credit to the original author(s) and the source, provide a link to the Creative Commons license, and indicate if changes were made. The images or other third party material in this article are included in the article's Creative Commons license, unless indicated otherwise in a credit line to the material. If material is not included in the article's Creative Commons license and your intended use is not permitted by statutory regulation or exceeds the permitted use, you will need to obtain permission directly from the copyright holder. To view a copy of this license, visit http://creativecommons.org/licenses/by/4.0/. 
By employing a BOCDA with optimized time gates and an unbalanced Mach-Zehnder delay line ${ }^{33}$, a vibration measurement with a frequency of $200 \mathrm{~Hz}$ and $10-\mathrm{cm}$ spatial resolution in a $20-\mathrm{m}$ measurement range is realized at a 1 $\mathrm{kHz}$ sampling rate. The sampling rate can be further improved to $5 \mathrm{kHz}$ based on random accessibility and the use of a lock-in amplifier ${ }^{34}$. An improved BOCDR scheme ${ }^{20}$ is implemented by utilizing a high-speed voltage-controlled oscillator to convert the BFS to a phase delay, resulting in a $100-\mathrm{kHz}$ sampling rate at a single point and $100-\mathrm{Hz}$ repetition rate at 1000 points with 10 times averaging. In the BOCDA/BOCDR techniques, the high-speed sampling rate for dynamic strain at a single point is obtained at the expense of distribution due to the slow sweeping peak location process, which requires priori information for the strain location.

Remarkably, BOTDA has been widely studied because of its superior performance for long-distance-distributed measurement ${ }^{27,35-38}$, wide-strain dynamic range ${ }^{12,31}$, and high-speed sampling rate $\mathrm{r}^{12,30,31,39-41}$. In a conventional BOTDA system, the distributed BGS is reconstructed by injecting a high-power pump pulse to interrogate the localstimulated Brillouin scattering (SBS) and then sweeping the continuous probe wave frequency over a wide spectral range; such a process imposes the main limiting factor on the sampling rate ${ }^{42}$. To date, BOTDA for dynamic measurement is classified into three categories: frequencycomb-based sweep-free scheme $e^{43-47}$, slope-assisted scheme ${ }^{30,31,39,42,48,49}$, and fast-frequency sweeping scheme ${ }^{50,51}$. The sweep-free scheme makes use of a frequency comb to demodulate the BGS; however, there is a trade-off between the spatial resolution and the frequency interval for the comb, with the spatial resolution usually a few tens of meters. In the slope-assisted scheme, the frequency detuning between pump and probe waves is set at the middle of the slopes for the BGS ${ }^{39,40,42}$, Brillouin phaseshift spectrum $^{30,52}$ or their ratio spectrum ${ }^{31,49}$, so that a single pump pulse can demodulate the distributed BFS along the fiber. It is noted that the sampling rate is only limited by the length of the FUT without averaging, but the dynamic range is restricted by the linewidth of the BGS. Several improved schemes have been introduced to improve the dynamic range to $5000 \mu \varepsilon$ by use of the frequency-agile technique $\mathrm{e}^{12,31,53}$ at the expense of the sampling rate. The last scheme is realized by compressing the frequency switching time for the probe wave. A $100-\mathrm{Hz}$ vibration measurement in a $100-\mathrm{m}$ FUT based on a fast BOTDA $^{50}$ is acquired by the frequency-agile technique to quickly switch 100 scanning frequencies for the probe wave corresponding to 100 synchronous pump pulses, resulting in a sampling rate of $\sim 10 \mathrm{kHz}$, with the distributed BGS tailored from the time trace. Obviously, the sampling rate for this scheme depends on both the length of the FUT and the number of scanning frequencies. Recently, a frequency- sweep pulsed BOTDA ${ }^{51}$ was implemented to obtain a Lorentzian-shaped correlated gain in the time domain by linear-frequency modulation of two 1.0- $\mu$ s pulses as pump and probe pulses coupled with a point $(20 \mathrm{~m})$ dynamic strain measurement with a sampling rate of $10 \mathrm{kHz}$ obtained at the end of $10 \mathrm{~km}$. Although a distributed dynamic measurement can be realized through adjusting the delay between the probe and pump pulses, the effective sampling rate is divided by the sensing point number.

In this paper, we theoretically and experimentally report a novel single-shot BOTDA for distributed ultrafast measurement based on optical chirp chain (OCC), named OCC-BOTDA, where the probe wave is frequency modulated into short optical chirp segments and then cascaded into an OCC. Only a single-shot pump pulse is required to recover the distributed BGS along the OCC probe wave when the local BFS is covered by the wide-frequency span between the pump pulse and the OCC probe wave. The maximum sampling rate can reach the order of $\mathrm{MHz}$, which is only restricted by the length of the FUT. The dynamic strain range can be readily tuned by setting the frequency span. We further test the distributed ultrafast performance of this system: a periodic mechanical vibration is monitored with a $25-\mathrm{kHz}$ sampling rate; a mechanical shock measurement is designed by rapidly stretching a 2-m fiber section via knocking a rotating mechanism, which is captured at a sampling rate of $2.5 \mathrm{MHz}$; a switch event is designed to simulate a fast $20-\mathrm{MHz}$ BFS change, which is recorded at a sampling rate of $6.25 \mathrm{MHz}$.

\section{Materials and methods Operation principle}

A typical BOTDA system is based on a "pump-probe" scheme ${ }^{2,28,31,54-56}$ such that the power transfer from the optical pump pulse to the continuous probe wave occurs through SBS when their frequency difference is set near the BFS of the FUT. The evolution of these two waves (indicated by $A_{\mathrm{p}}$ and $A_{\mathrm{s}}$, respectively) and an acoustic wave (indicated by $Q$ ) is described by the following coupled-wave equations ${ }^{2,57}$

$$
\begin{gathered}
\frac{\partial A_{\mathrm{p}}}{\partial z}+\frac{n}{c} \frac{\partial A_{\mathrm{p}}}{\partial t}=\frac{i \omega \gamma_{\mathrm{e}}}{2 n c \rho_{0}} Q A_{\mathrm{s}} \\
\frac{\partial A_{\mathrm{s}}}{\partial z}-\frac{n}{c} \frac{\partial A_{\mathrm{s}}}{\partial t}=-\frac{i \omega \mathrm{e}_{\mathrm{e}}}{2 n c \rho_{0}} Q^{*} A_{\mathrm{p}} \\
\left(\Gamma_{\mathrm{B}}-2 i \Omega\right) \frac{\partial Q}{\partial t}+\left(\Omega_{\mathrm{B}}^{2}-\Omega^{2}-i \Omega \Gamma_{\mathrm{B}}\right) Q=\varepsilon_{0} \gamma_{\mathrm{e}} q^{2} A_{\mathrm{p}} A_{\mathrm{s}}^{*}
\end{gathered}
$$

where $\omega \equiv 2 \pi \cdot v_{\mathrm{p}} \approx 2 \pi \cdot v_{\mathrm{s}}$ is the angular frequency of the optical waves, $n$ is the refractive index of the FUT core, $c$ is the speed of light in the vacuum, $\gamma_{\mathrm{e}}$ is the electrostrictive coefficient, $\rho_{0}$ denotes the mean density of the FUT core, $\varepsilon_{0}$ is the vacuum permittivity, and $\Gamma_{\mathrm{B}}=1 / \tau_{\mathrm{p}}$ is the Brillouin gain linewidth. $\Omega=2 \pi\left(v_{\mathrm{p}}-v_{\mathrm{s}}\right), q=k_{\mathrm{p}}+k_{\mathrm{s}} . \Omega_{\mathrm{B}}=2 \pi \cdot f_{\mathrm{BFS}}$, where $f_{\mathrm{BFS}}=2 n v_{\mathrm{p}} V_{\mathrm{a}} / c$ is the local BFS of the FUT and $V_{\mathrm{a}}$ is 


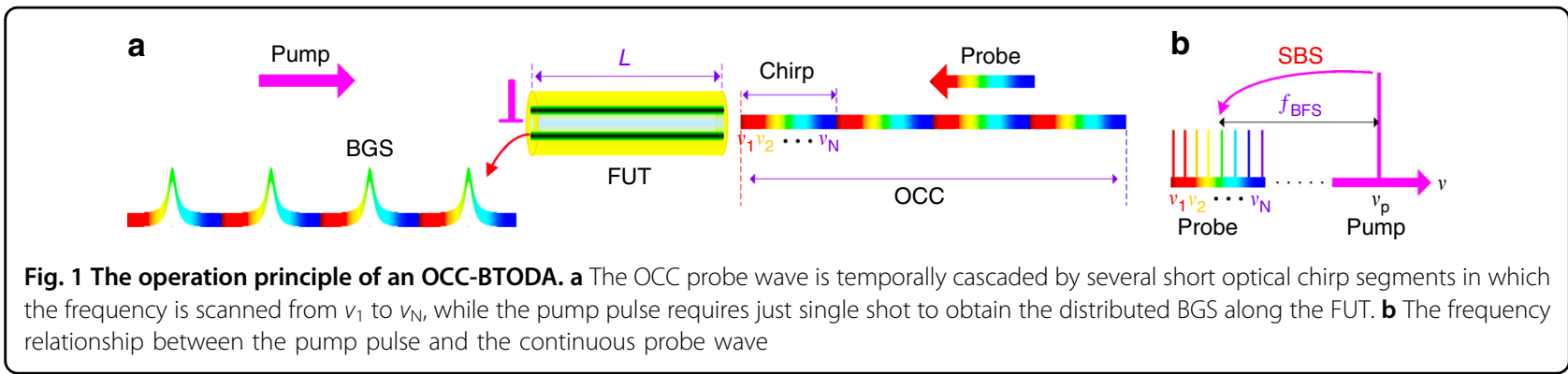

the speed of the moving acoustic wave. Generally, the value of the BFS is $\sim 11 \mathrm{GHz}$ for a single-mode fiber at the communication wavelength of $1550 \mathrm{~nm}^{2,57}$.

The operation principle for the OCC-BOTDA is illustrated in Fig. 1a. The optical probe wave is frequency modulated from $v_{1}$ to $v_{\mathrm{N}}(\mathrm{N}$ is the frequency number $)$ in a few tens of nanoseconds, resulting in a short optical chirp segment, which can be regarded as the temporal compression of the traditional sweeping frequency method. Then, the probe wave is composed of several short optical chirp segments, which are referred to as the optical chirp chain (OCC). It is noted that the OCC has two types of on-link modes: one is the sawtooth mode (two adjacent optical short-chirp segments are cascaded by a head-to-tail cohesion), and the other is the triangular mode (two adjacent optical short-chirp segments are cascaded by a head-to-head or tail-to-tail cohesion). Similar to typical BOTDA systems, the pump wave is shaped into a pulse pattern, with a width that is narrower than the duration of the short optical chirp segment. The OCC probe wave and the pump pulse are injected into an FUT in the opposite direction. When the frequency difference between the pump pulse and the OCC probe wave matches the BFS for the FUT (Fig. 1b), the BGS can be recovered along the short optical chirp segment in the time domain due to the Brillouin amplification.

The OCC-BOTDA system is numerically calculated based on Eq. (1), with the simulation results corresponding to the two chirp modes (magenta line), i.e., "sawtooth" mode and "triangular" mode shown in Fig. 2a, b, respectively. Here, the duration of the short optical chirp segment is set at $20 \mathrm{~ns}$, with $300 \mathrm{MHz}$ of frequency coverage, while the width of the pump pulse is set at $10 \mathrm{~ns}$. A 10-m-long fiber with $f_{\mathrm{BFS}}=10.70 \mathrm{GHz}$ and $\Gamma_{\mathrm{B}} / 2 \pi=30$ $\mathrm{MHz}$ is used. It is clearly illustrated that the intrinsic BGSs (black dashed-dotted line) along the fiber describes a symmetrical Lorentzian profile as the frequency of the OCC is scanned from $10.550 \mathrm{GHz}$ to $10.845 \mathrm{GHz}$ in every 2 -m segment. Compared with the intrinsic BGS, in both the "sawtooth" mode and "triangular" mode, the simulated BGS exhibits an asymmetrical and broadened profile, while its peak shows a time delay of $\sim 2.7 \mathrm{~ns}$ (or position shift) corresponding to a frequency shift of $\sim 41$ MHz. Such a phenomenon arises from the transient SBS interaction, i.e., the hysteresis effect due to Brillouin amplification with respect to the acoustic wave excitation. As shown in Fig. 2c, the peaks of the intrinsic BGS and the derivative of the simulated BGS (green line) show complete coincidence, which indicates that the growth rate of the acoustic wave reaches a maximum for the resonant frequency and then decreases due to the increased detuning from the BFS; subsequently, the measured BGS exhibits a time delay with respect to the intrinsic case.

Note that a "ghost peak" appears at the frequency discontinuity position in the "sawtooth" mode in Fig. 2a due to the generation of an equivalent frequency near the BFS when the frequency is decreased from the highest frequency component $v_{\mathrm{N}}$ to the lowest frequency component $v_{1}$; the "ghost peak" disappears in the "triangular" mode in Fig. $2 \mathrm{~b}$ due to the smooth convergence for the frequency in the link point between two short optical chirp segments. In the following experiment, the "sawtooth" mode OCC probe wave is adopted, since the demodulation process for the local BFS in the "sawtooth" mode is simpler than in the "triangular" mode.

\section{Sampling rate and spatial resolution}

In contrast to the time-consuming process of frequency sweeping for traditional BOTDA, the distributed BGS along the FUT based on the OCC-BOTDA can be rapidly demodulated by only injecting a single-shot pump pulse into the fiber. Therefore, the maximum sampling rate of this scheme is given by

$$
f_{\mathrm{Sa}}=\frac{1}{N_{\mathrm{ave}} \cdot T}
$$

where $N_{\text {ave }}$ is the number of averages, $T=2 n L / c$ is the round-trip time for the sensing fiber with a length of $L$. It can be seen that without averaging, the maximum sampling rate is only limited by the FUT length.

The spatial resolution for this scheme is restricted by the duration of the short optical chirp segment $\Delta T$ and is given by

$$
\Delta z_{\mathrm{SR}}=\frac{c \Delta T}{2 n}
$$


a

Time (ns)

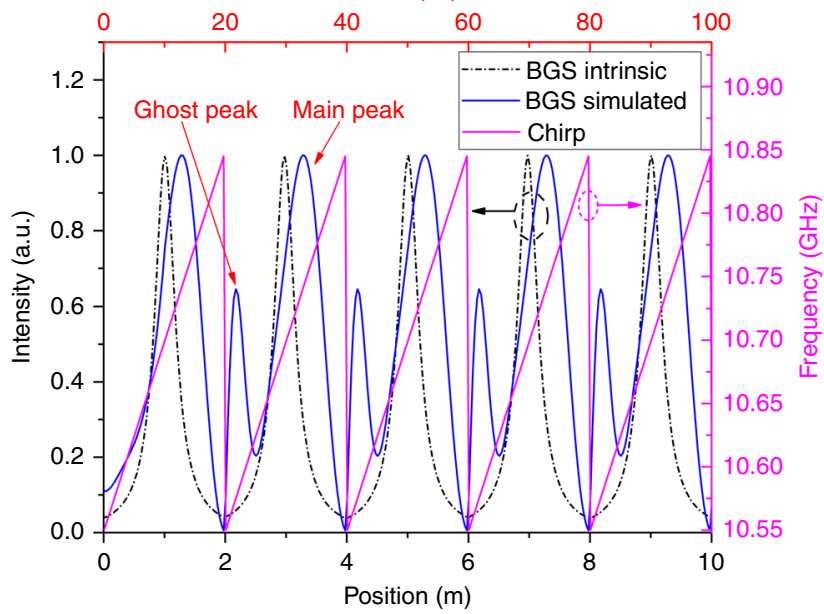

b

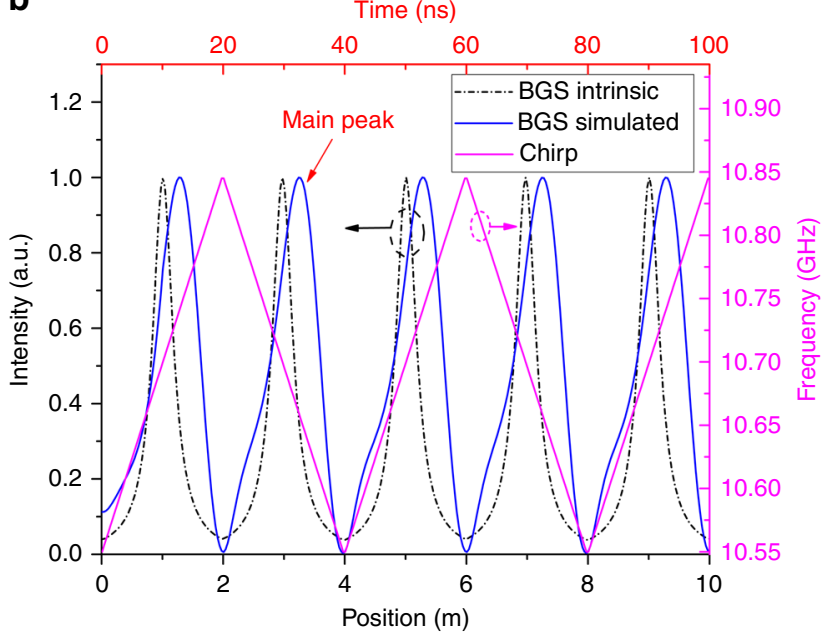

C

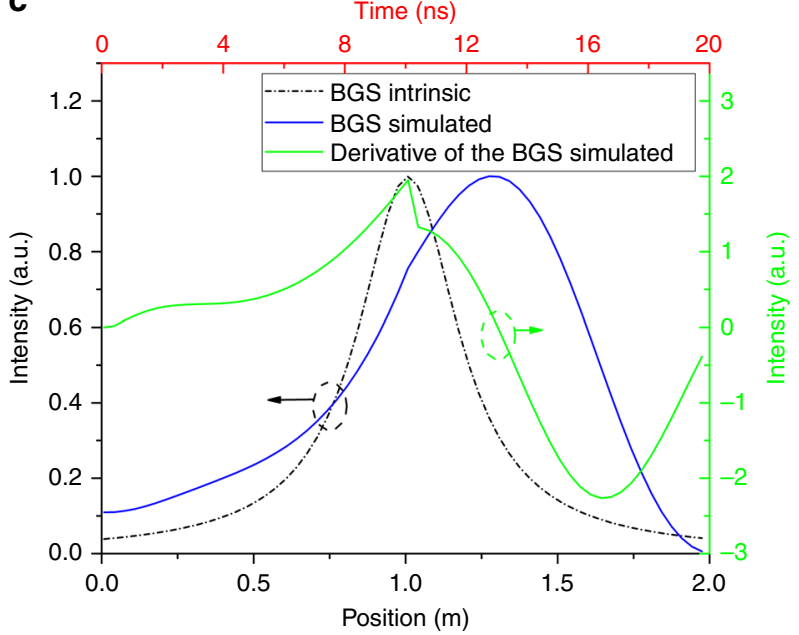

Fig. 2 Simulation of the OCC-BOTDA. a The distributed BGSs along the fiber for the intrinsic case (black dashed-dotted line), "sawtooth" mode (blue line), and distributed chirp frequency (magenta line). b The distributed BGSs along the fiber for the intrinsic case (black dashed-dotted line), "triangular" mode (blue line), and distributed chirp frequency (magenta line). c The intrinsic BGS (black dashed-dotted line), simulated BGS (blue line), and derivative of the simulated BGS (green line) within the first $2 \mathrm{~m}$ 


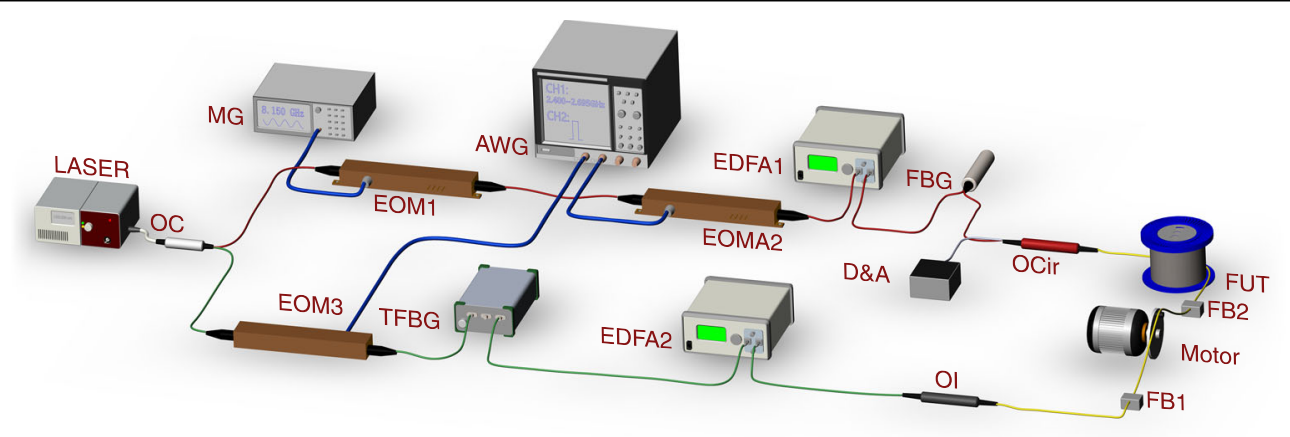

Fig. 3 Experimental configuration for the OCC-BTODA system. MG microwave generator, EOM electro-optic modulator, AWG arbitrary-waveform generator, EDFA erbium-doped fiber amplifier, FUT fiber under test, TFBG tunable fiber Bragg grating, OC optical coupler, Ol optical isolator, OCir optical circulator, D\&A photodetector and data acquisition modules, FB fixed base. Note that the polarization for all the optical devices is aligned along the slow axis to eliminate polarization noise and fading

\section{Experimental setup}

Figure 3 shows the experimental setup used to test the ultrafast performance of the OCC-BOTDA scheme. The frequency-agile technique involves generation of fastfrequency-changing microwaves by an AWG with a high bandwidth and high digital sampling rate, in which the output can be reconfigured by editing the AWG control program. The frequencies should satisfy the equation ${ }^{53}$

$$
f_{\mathrm{AWG}}(t)=f_{1}+\eta \cdot t
$$

where $f_{1}$ is the initial frequency. $\eta=\Delta f / \Delta T$ is the frequency-changing rate, $\Delta f$ is the frequency span, and $\Delta T$ is the duration of the short optical chirp segment. Due to the bandwidth limitation for AWG in our laboratory, we chose the dual modulation ${ }^{53}$ to implement a widefrequency span between the OCC probe wave and the pump pulse, which can cover the BFS of the FUT.

An optical fiber laser with a narrow linewidth of $\sim 10 \mathrm{kHz}$ was used as the light source, in which the output parameters were set at $1550 \mathrm{~nm}$ and $100 \mathrm{~mW}$. Subsequently, the $90 \%$ branch (red line) for the optical coupler was selected to be chopped for the optical pulse as the pump wave. The light was frequency modulated using an electro-optic modulator1 in the carrier-suppressed regime, which is driven by an 8.15$\mathrm{GHz}$ sinusoidal microwave output from a microwave generator. Then, the frequency-modulated light is intensity modulated using EOM2 driven by the $\mathrm{CH} 2$ of the AWG into a 10-ns optical pulse. After amplification to $1 \mathrm{~W}$ by an erbium-doped fiber amplifier1, an optical pulse with a higher-frequency sideband was selected through an optical fiber Bragg grating (FBG) filter. Then, the optical pulse was injected into the FUT through an optical circulator.

The lower branch with $10 \%$ component was used to generate an OCC for use as a probe wave. The light was frequency downshifted using EOM3 driven by the $\mathrm{CH} 1$ of AWG and a tunable FBG. Note that the output of $\mathrm{CH} 1$ was temporally cascaded by the sinusoidal microwave signals with a frequency span from 2.4 to $2.695 \mathrm{GHz}$ and a frequency step of $5 \mathrm{MHz}$. As a result, a short optical chirp segment was generated with a width of $20 \mathrm{~ns}$ and frequency-changing rate of $\eta=15 \mathrm{MHz} / \mathrm{ns}$. By using the "sawtooth" mode, the OCC probe wave was assembled and then amplified by EDFA2 to $\sim 80 \mu \mathrm{W}$. Hence, both the pump pulse and the continuous probe wave were launched into the FUT. Finally, the Brillouin signal (i.e., amplified OCC-probe wave) was received by a D\&A module at a sampling rate of $5 \mathrm{GS} / \mathrm{s}$. To eliminate polarization noise and fading, the polarization of all optical devices was aligned along the slow axis, with a polarization maintaining fiber (PMF) with a BFS of $10.705 \mathrm{GHz}$ used as the FUT. A photodetector with a bandwidth of $350 \mathrm{MHz}$ and a response time of $2 \mathrm{~ns}$ in the D\&A module was used to detect the OCC-probe wave.

\section{Results and discussions}

Static BGS measurement and twice-correlation algorithm

The measured time traces of the Brillouin signal of the OCC-BOTDA system are shown in Fig. 4a. The data clearly show a BGS with a main peak and a ghost peak that is found to occur every $20 \mathrm{~ns}$ (corresponding to a spatial resolution of $2 \mathrm{~m}$ ), which agrees well with the simulation results, as plotted in Fig. 2. Compared with the BGS (black line) for a strain change of $\Delta \varepsilon=0.0 \mu \varepsilon$, the BGS (magenta line within the blue wireframe) for a strain change $\Delta \varepsilon=700$ $\mu \varepsilon$ is time-shifted. Subsequently, a zoom-in view for the blue wireframe is shown in Fig. $4 \mathrm{~b}$ as the strain changes from $\Delta \varepsilon=0.0 \mu \varepsilon$ to $\Delta \varepsilon=700 \mu \varepsilon$, with the horizontal axis label converted from a time value to a frequency value. It is clearly shown that the central frequency of the main peak initially shifts by $\sim 42 \mathrm{MHz}$ with respect to the local BFS at $\sim 10.705 \mathrm{GHz}$ at room temperature. The time (or frequency) of the main peak is evidently up-shifted as the strain change is increased.

However, it is very difficult to precisely calculate the BFS by typical Lorentzian or Gaussian fitting the main 

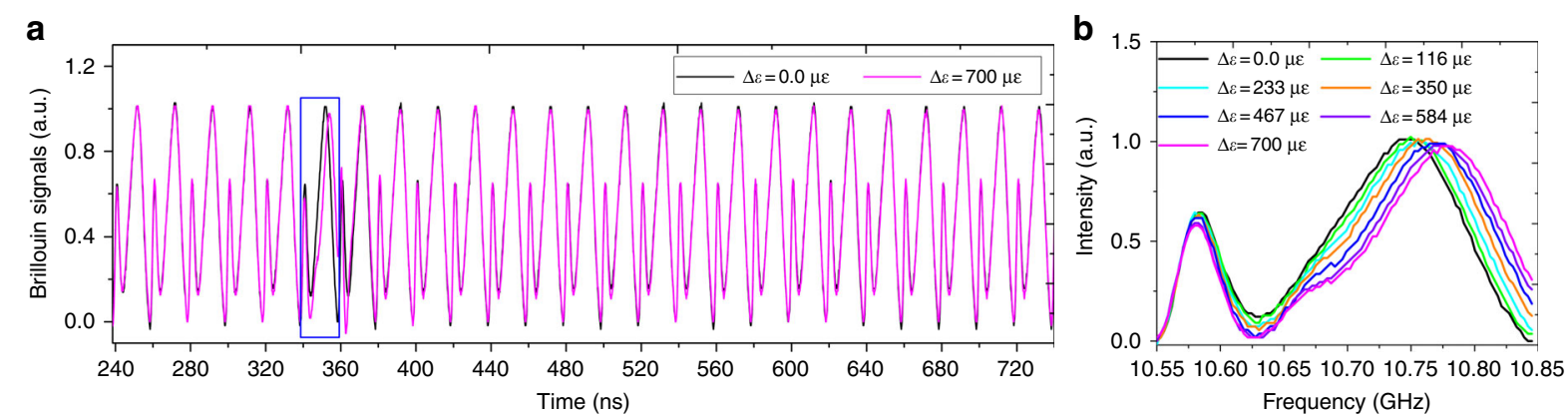

Fig. 4 The static distributed BGS measurement based on the proposed OCC-BOTDA. a The time traces for the Brillouin signals for a strain change of $\Delta \varepsilon=0.0 \mu \varepsilon$ and $\Delta \varepsilon=700 \mu \varepsilon$. b The zoom-in view for the BGSs for a strain change from $\Delta \varepsilon=0.0 \mu \varepsilon$ to $\Delta \varepsilon=700 \mu \varepsilon$ in the blue box. Averaging is carried out 200 times
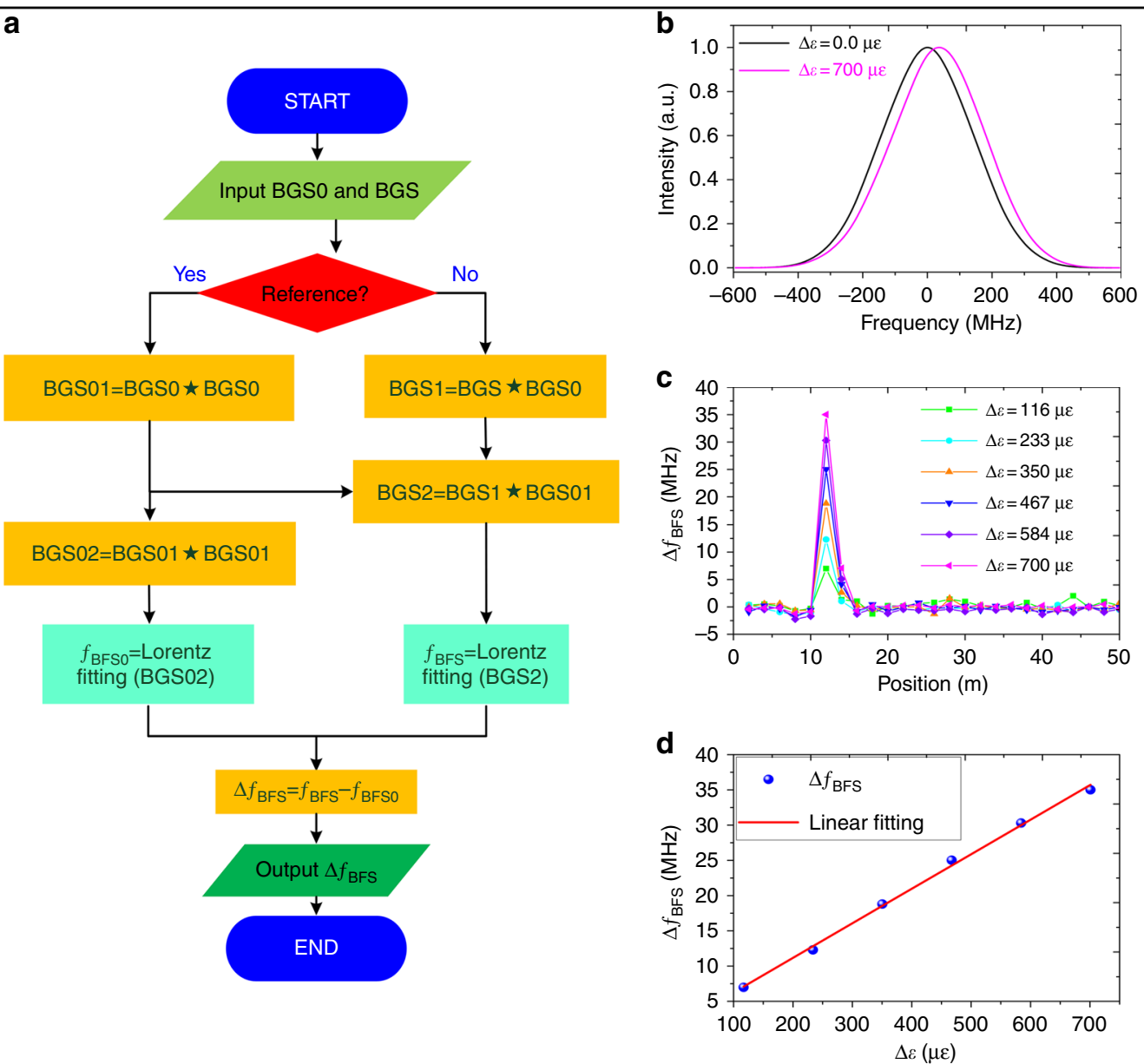

Fig. 5 The strain change demodulation process. a The demodulation flow chart based on a twice-correlation algorithm; $\star$ represents the crosscorrelation operator. $\mathbf{b}$ The reference BGS and the random BGS are processed by this algorithm, resulting in two symmetrical profiles. $\mathbf{c}$ The demodulated BFS changes along the 50-m PMF. $\mathbf{d}$ Change in BFS versus change in strain

peak of the BGS due to its asymmetrical profile. Here, we propose a twice-correlation algorithm not only to make the measured BGS more symmetrical, but also to improve the signal-to-noise ratio $(\mathrm{SNR})^{58,59}$. Based on the intrinsic mathematical property of the correlation function, this algorithm can be used to realize two functions. One is the symmetric correlation peak generated by the auto- or cross-correlation between the BGSs, which enables a 


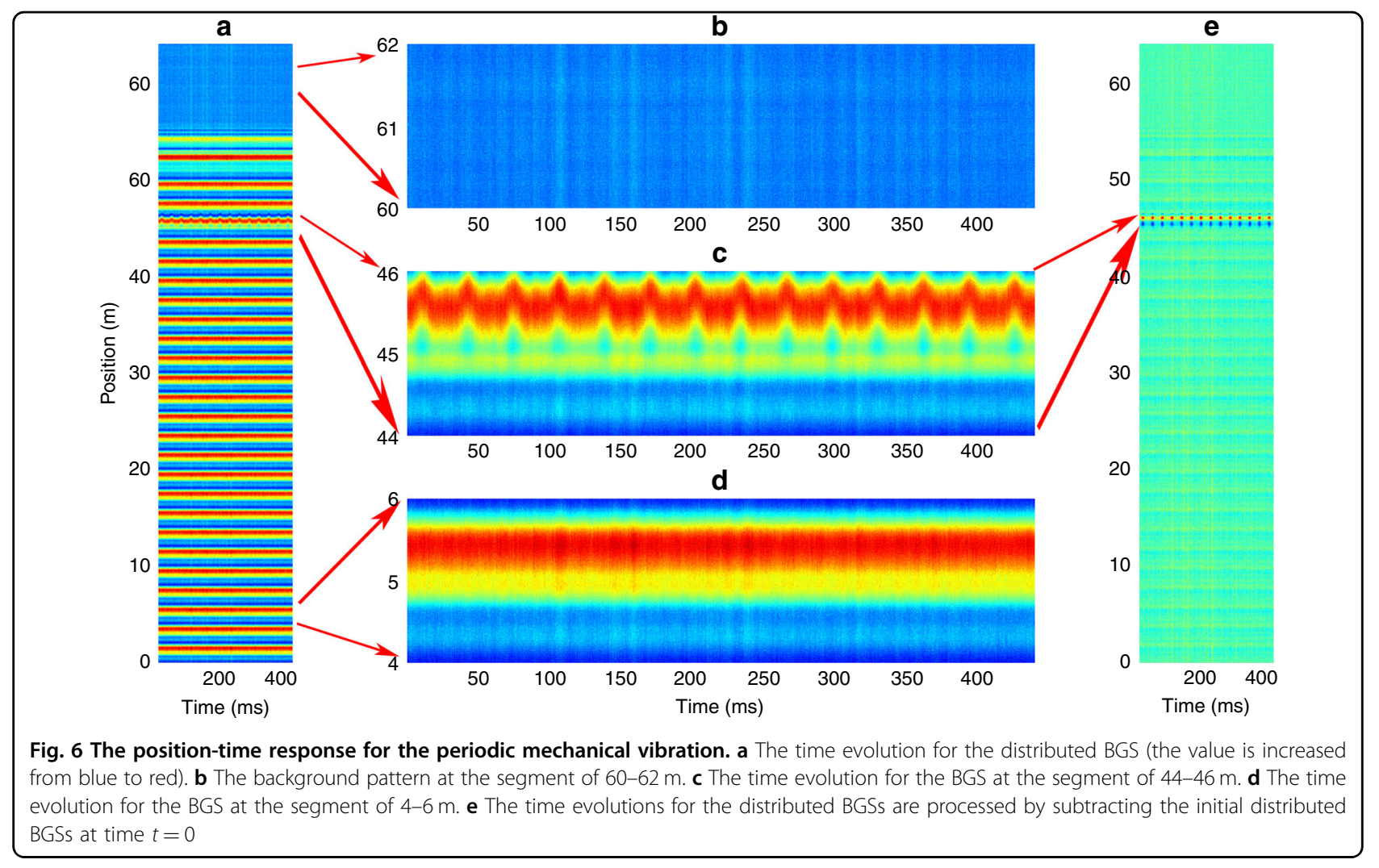

much easier precise calculation for the main peak location. The other function is the position or frequency shift of the correlation peaks for different strains, corresponding to changes in BFS or strain.

The demodulation flow chart for this algorithm is shown in Fig. 5a. First, a measured BGS in the loose region is selected as the reference BGS0, and then, its first autocorrelation peak BGS01 and second autocorrelation peak BGS02 (black line in Fig. 5b) are computed. The twice-correlation results for the measured BGSs are given by

$$
\mathrm{BGS} 2=(\mathrm{BGS} \star \mathrm{BGSO}) \star \mathrm{BGS} 01
$$

where $\star$ represents the cross-correlation operator. It can be seen that both correlation peaks BGS02 and BGS2 (e.g., magenta line for $\Delta \varepsilon=700 \mu \varepsilon$ ) show more symmetrical profiles and higher SNR compared to the original BGSs in Fig. 4b. Next, the correlation peaks BGS02 and BGS2 are Lorentzian or Gaussian fitted to calculate their central frequency $f_{\mathrm{BFS} 0}$ and $f_{\mathrm{BFS}}$. Finally, the output of this flow chart is the BFS change

$$
\Delta f_{\mathrm{BFS}}=f_{\mathrm{BFS}}-f_{\mathrm{BFS} 0}
$$

As a result, the distributed BFS changes for different strain measurements are shown in Fig. 5c; the data show that the demodulated BFS change in the strain segment at $10-12 \mathrm{~m}$ is clearly migrated away from a value of approximately zero in the non-strain segment. The strain dependences for the demodulated BFS changes are plotted in Fig. 5d, which reveals a good linearity with a fitting strain coefficient of $0.049 \mathrm{MHz} / \mu \varepsilon$.

\section{Dynamic strain measurements}

To test the dynamic measurement performance, three different types of dynamic strain are established as follows:

\section{Periodic mechanical vibration measurement}

A 2-m segment located at a position of $44-46 \mathrm{~m}$ for a $50-\mathrm{m}$ FUT is driven by an electro-motor to induce a periodic mechanical vibration for the test. The frequencychanging rate $\eta$ is set to $20 \mathrm{MHz} / \mathrm{ns}$, while the duration for the short optical chirp segment is set to $20 \mathrm{~ns}$. For data acquisition, the original measured time trace for the Brillouin signal with no averaging can be collected via the fast-frame mode while setting the sampling rate to $25 \mathrm{kHz}$ (corresponding to a period of $40 \mu \mathrm{s}$ ).

According to the order of frame and time, the time trace is reshaped with frame length $N_{\text {seg }}=3200(>2 n L / c \cdot 5 \mathrm{GS} / \mathrm{s})$, forming a $3200 \times 11000$ array. The time evolution for the distributed BGS is shown in Fig. 6a. The segment of 60-62 m without signal, as shown in Fig. 6b, corresponds to a region where no sensing fiber is present. As shown in Fig. $6 \mathrm{c}$, the BGSs at the vibrated segment of $44-46 \mathrm{~m}$ are 

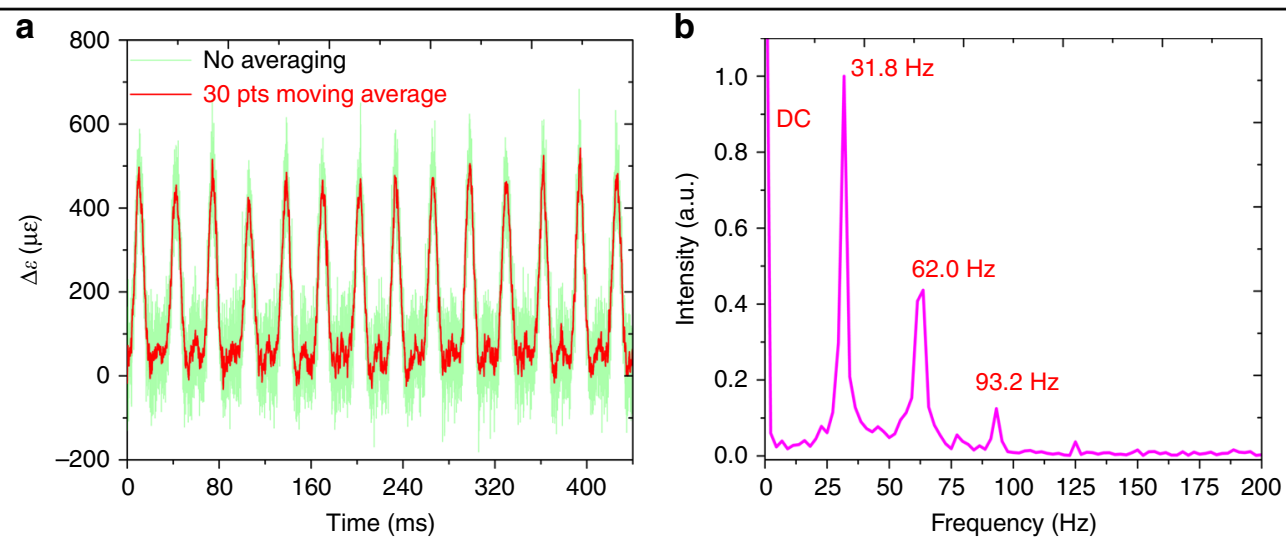

Fig. 7 The time and frequency distribution of the periodic mechanical vibration. a The time evolution for the strain changes (green line) with no averaging, which represents the waveform for the periodic mechanical vibration, and 30-points moving average result (red line). $\mathbf{b}$ The frequency spectrum of the vibration

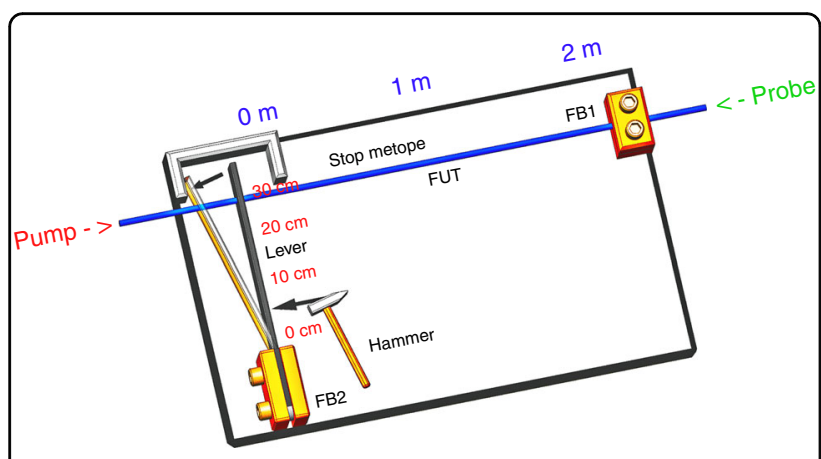

Fig. 8 Schematic diagram for the mechanical shock measurement

position-shifted over time, while the BGSs at the loose segment of 4-6 m remain unchanged in Fig. 6d. Subsequently, by subtracting the initial distributed BGSs at time $t=0$, the vibration position span and periodic patterns are rapidly processed, which can then be clearly located as demonstrated in Fig. 6e, which is suitable for real-time identification of vibration locations. The green line in Fig. 7a shows the time evolution for the strain change at the segment of 44-46 m demodulated by the "twice-correlation algorithm," with the red line showing the result of a 30-points moving average, which corresponds to the waveform of the periodic mechanical vibration. The frequency spectrum for this waveform is obtained through fast Fourier transform, as shown in Fig. 7b, which contains a fundamental frequency component of $31.8 \mathrm{~Hz}$ with harmonics of 62.0 and $93.2 \mathrm{~Hz}$.

\section{Mechanical shock measurement}

A mechanical shock measurement is designed, as shown by the schematic diagram in Fig. 8. A 2-m segment of a $10-\mathrm{m}$ FUT is selected to be stretched. The two end points for this section are fixed onto FB1 and the free end of a lever. In this experiment, through rapidly impacting the lever by a hammer, the free end of the lever will be rotated around FB2 so that the 2-m fiber section is quickly stretched. Meanwhile, this mechanical shock is captured by the proposed OCC-BOTDA system in which the sampling rate is set at $2.5 \mathrm{MHz}$ (corresponding to a period of $400 \mathrm{~ns}$ ). The frequency-changing rate $\eta$ is $15 \mathrm{MHz} / \mathrm{ns}$, while the duration for the short optical chirp segment is set to $20 \mathrm{~ns}$.

The time evolutions for the BGSs in this section are first processed by the "twice-correlation algorithm," with the result plotted in Fig. 9a. The demodulated strain change induced by mechanical shock is shown in Fig. 9b, where the green line shows the original result with no averaging and the red line shows the result obtained for a 30-points moving average. A shock time of $\sim 250 \mu$ s with a strain change of $\sim 800 \mu \varepsilon$ is demonstrated from the no-strain state at time $t=\sim 400 \mu \mathrm{s}$ to the strain state at time $t=\sim 750 \mu \mathrm{s}$. This result shows tremendous potential for capturing fast processes, e.g., an explosion process. The standard deviation for the result is $\sim 120 \mu \varepsilon$ with no averaging, while it decreases to $35 \mu \varepsilon$ for 30 -points moving averaging since the power magnification of EDFA2 decreases as the repetition rate of the pump pulse increases to the order of a MHz.

\section{Switch event demo}

A switch event is carried out to demonstrate the ultrafast acquisition capability of the OCC-BOTDA system. The frequency-changing rate $\eta$ and the duration for the short optical chirp segment are set to $15 \mathrm{MHz} / \mathrm{ns}$ and 20 ns, respectively, as done previously. Its notion can be summarized by noting that the microwave frequency agility spans every adjacent optical chirp segment are set at $2.400-2.695 \mathrm{GHz}$ and $2.380-2.675 \mathrm{GHz}$, resulting in a frequency shift of $20 \mathrm{MHz}$ (i.e., a strain change of $\sim 408$ 

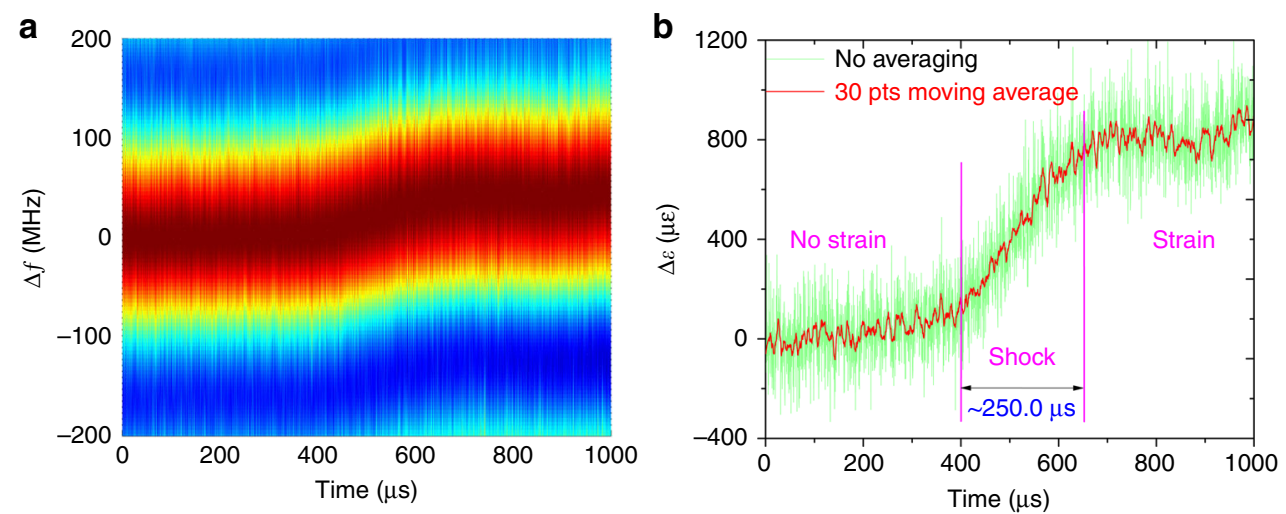

Fig. 9 Time-frequency response for the mechanical shock measurement. a The BGS over time processed by the "twice-correlation algorithm" and $\mathbf{b}$ its strain change over time with no averaging (green line) and the result obtained for a 30-points moving average (red line)
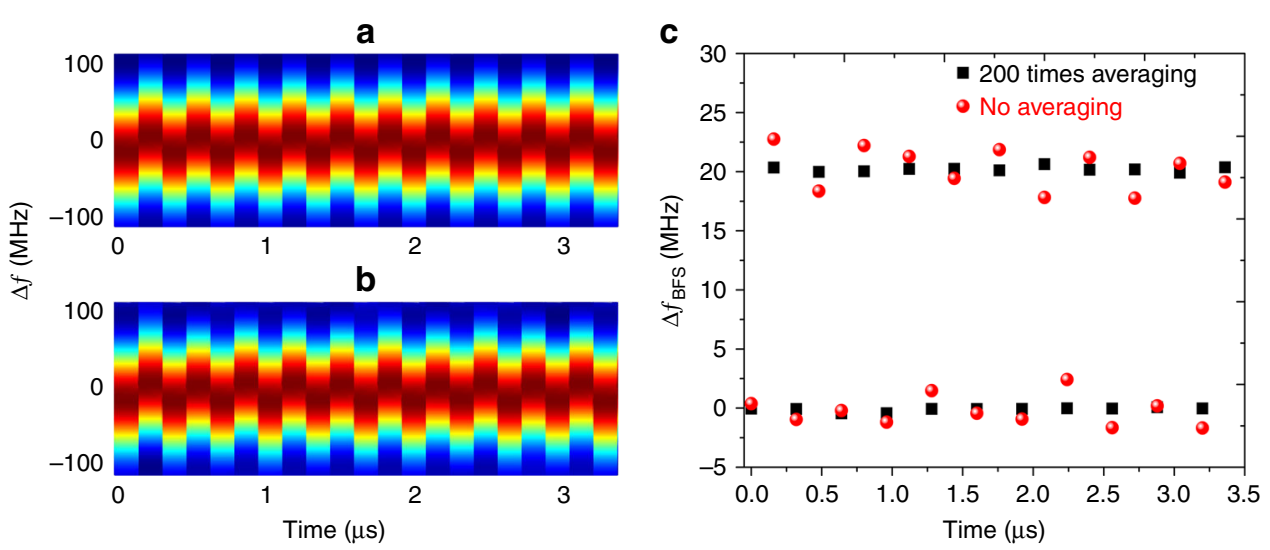

Fig. 10 The time-frequency response for the switch event. The BGS over time is processed by the "twice-correlation algorithm" for a averaging 200 times and $\mathbf{b}$ no averaging. c The BFS changes over time for averaging 200 times (black scatter) and no averaging (red scatter)

$\mu \varepsilon)$, which can be used to simulate a quick BFS change over time. The repetition rate for the pump pulse can reach up to $6.25 \mathrm{MHz}$ (corresponding to a period of 160 ns).

The time evolution for the BGSs is processed by the "twice-correlation algorithm" at 200 times averaging and no averaging, as shown in Fig. 10a, b, respectively. Their BFS changes over time are shown in Fig. 10c, where the lower and the upper scatter sequences correspond to the frequency-agility spans of $2.400-2.695 \mathrm{GHz}$ and $2.380-2.675 \mathrm{GHz}$, respectively. The frequency difference between the two scatter sequences is $\sim 20 \mathrm{MHz}$, with the error for averaging 200 times and no averaging lying within $\pm 0.5 \mathrm{MHz}$ and $\pm 2.5 \mathrm{MHz}$, respectively.

\section{Conclusions}

In this work, an OCC-BOTDA scheme was theoretically proposed, numerically simulated, and experimentally verified for distributed ultrafast measurement with a maximum sampling rate of $6.25 \mathrm{MHz}$. The OCC probe wave is composed of several short optical chirp segments, which are generated through frequency modulation by use of the frequency-agile technique. When the frequency span between the OCC probe wave and the pump pulse overlaps the BFS for the sensing fiber, the distributed BGS along the fiber is revealed via the OCC probe wave in the time (or position) domain using only a single-shot pump pulse. The sampling rate for this proposed OCC-BOTDA is only restricted by the fiber length.

The performance for this proposed OCC-BOTDA is limited by the trade-offs among the frequency span, spatial resolution, and SNR. The effective scanning range is less than the frequency span due to the influence of a "ghost peak" and broadened BGS profile. As the frequency span increases for a wide-dynamic range, the SBS interaction region will be compressed, resulting in recovery of the BGS over a narrow region with a deteriorating SNR. The relatively lower SNR in this work may be due to the following 
factor: the AWG used in this work is composed by syncing a single output channel AWG with a high-sampling rate to an arbitrary-function generator with a lower-sampling rate, which results in electrical signal jitter with a root-meansquare of $\sim 100$ ps between the pump pulse and the OCC probe wave. For future work, a high-performance dualchannel AWG can be used to generate the pump pulse and the OCC probe wave to increase the SNR.

Additionally, the D\&A module can be followed by a field-programmable gate array (FPGA) to realize a realtime response. We believe that the proposed OCC-BOTDA can provide dynamic or real-time distributed measurement for many potential applications, e.g., monitoring of large infrastructure and capturing an explosion process.

\section{Acknowledgements \\ This work was supported by the National Key Scientific Instrument and Equipment Development Project of China (2017YFF0108700) and National Natural Science Foundation of China (61575052). We like to express our gratitude to P.B. Xu and T.F. jiang for their suggestion in the primary experiment and P.L. Tong and L.W. Sheng for their contribution to the diagrams for the experimental setup.}

\section{Author details}

${ }^{1}$ National Key Laboratory of Science and Technology on Tunable Laser, Harbin Institute of Technology, 150001 Harbin, China. ${ }^{2}$ Department of Optoelectronic Information, Science and Engineering, Harbin University of Science and Technology, 150080 Harbin, China. ${ }^{3}$ School of Civil Engineering, Harbin Institute of Technology, 150001 Harbin, China. ${ }^{4}$ Fiber Optics Group, Department of Physics, University of Ottawa, Ottawa, ON K1N 6N5, Canada

\section{Conflict of interest}

The authors declare no conflict of interest.

Received: 13 September 2017 Revised: 13 April 2018 Accepted: 7 May 2018 Accepted article preview online: 11 May 2018

Published online: 11 July 2018

\section{References}

1. Bao, X. Y. \& Chen, L. Recent progress in distributed fiber optic sensors. Sensors 12, 8601-8639 (2012).

2. Bao, X. Y. \& Chen, L. Recent progress in brillouin scattering based fiber sensors. Sensors 11, 4152-4187 (2011).

3. Hotate K. Recent achievements in BOCDA/BOCDR. Proceedings of IEEE SENSORS 142-145 (IEEE, Valencia, Spain, 2014).

4. He, Z. Y., Liu, Q. W. \& Tokunaga, T. Ultrahigh resolution fiber-optic quasi-static strain sensors for geophysical research. Photon. Sens. 3, 295-303 (2013).

5. Mirhabibi, M., Negarestani, A., Bolorizadeh, M. A., Rezaei, M. R., \& Akhound, A. A new approach for radon monitoring in soil as an earthquake precursor using optical fiber. J. Radioanal. Nucl. Chem. 301, 207-211 (2014).

6. Zhang, W. T., Huang, W. Z., Li, L., Liu, W. Y. \& Li, F. High resolution strain sensor for earthquake precursor observation and earthquake monitoring. Proceedings of the 6th European Workshop on Optical Fibre Sensors (SPIE, Limerick, Ireland, 2016).

7. Leo, J., Low, K., Costa, J., Black, R. J. \& Park, Y. L. Fiber optically sensorized multi-fingered robotic hand. Proceedings of 2015 IEEE/RSJ International Conference on Intelligent Robots and Systems 1763-1768 (IEEE, Hamburg, Germany, 2015).

8. Xiao L. F. et al. Impedance control of a robot needle with a fiber optic force sensor. Proceedings of the 13th International Conference on Signal Processing 1379-1383 (IEEE, Chengdu, China, 2016).
9. Guo, J. J., Niu, M. X. \& Yang, C. X. Highly flexible and stretchable optical strain sensing for human motion detection. Optica 4, 1285-1288 (2017).

10. Zadok, A. et al. Random-access distributed fiber sensing. Laser Photonics Rev. 6 L1-L5 (2012).

11. Kurashima, T., Horiguchi, T. \& Tateda, M. Distributed-temperature sensing using stimulated Brillouin scattering in optical silica fibers. Opt. Lett. 15, 1038-1040 (1990).

12. Ba, D. X. et al. Distributed measurement of dynamic strain based on multislope assisted fast BOTDA. Opt. Express 24, 9781-9793 (2016).

13. Kurashima, T., Horiguchi, T. \& Tateda, M. Thermal effects of Brillouin gain spectra in single-mode fibers. IEEE Photonics Technol. Lett. 2, 718-720 (1990).

14. Niklès, M., Thévenaz, L. \& Robert, P. A. Simple distributed fiber sensor based on Brillouin gain spectrum analysis. Opt. Lett. 21, 758-760 (1996).

15. Bao, X., Webb, D. J. \& Jackson, D. A. 22-km distributed temperature sensor using Brillouin gain in an optical fiber. Opt. Lett. 18, 552-554 (1993).

16. Hotate, K. Fiber distributed brillouin sensing with optical correlation-domain techniques. Proceedings of Asia Communications and Photonics Conference (OSA, Shanghai, China, 2014).

17. Hotate, K. Arai, H. \& Song, K. Y. Range-enlargement of simplified brillouin optical correlation domain analysis based on a temporal gating scheme. SICE J. Control Meas. Syst. Integr. 1, 271-274 (2008).

18. Song, K. Y., He, Z. Y. \& Hotate, K. Distributed strain measurement with millimeter-order spatial resolution based on Brillouin optical correlation domain analysis. Opt. Lett. 31, 2526-2528 (2006).

19. Mizuno, Y., Zou, W. W., He, Z. Y. \& Hotate, K. Proposal of Brillouin optical correlation-domain reflectometry (BOCDR). Opt. Express 16, 12148-12153 (2008).

20. Mizuno, Y., Hayashi, N., Fukuda, H., Song, K. Y. \& Nakamura, K. Ultrahigh-speed distributed Brillouin reflectometry. Light Sci. Appl. 5, e16184 (2016).

21. Lee, H., Hayashi, N., Mizuno, Y. \& Nakamura, K. Slope-assisted Brillouin optical correlation-domain reflectometry: proof of concept. IEEE Photonics J. 8 6802807 (2016).

22. Mizuno, Y., He, Z. Y. \& Hotate, K. One-end-access high-speed distributed strain measurement with 13-mm spatial resolution based on Brillouin optical correlation-domain reflectometry. IEEE Photonics Technol. Lett. 21, 474-476 (2009).

23. Lee, H., Hayashi, N., Mizuno, Y. \& Nakamura, K. Operation of slope-assisted Brillouin optical correlation-domain reflectometry: comparison of system output with actual frequency shift distribution. Opt. Express 24, 29190-29197 (2016).

24. Manotham, S., Kishi, M., He, Z. Y. \& Hotate K. 1-cm spatial resolution with large dynamic range in strain distributed sensing by Brillouin optical correlation domain reflectometry based on intensity modulation. Proceedings Volume 8351, Third Asia Pacific Optical Sensors Conference (SPIE, Sydney, Australia, 2012)

25. Denisov, A., Soto, M. A. \& Thévenaz, L. Going beyond 1000000 resolved points in a Brillouin distributed fiber sensor: theoretical analysis and experimental demonstration. Light Sci. Appl. 5, e16074 (2016).

26. Li, W. H., Bao, X. Y., Li, Y. \& Chen, L. Differential pulse-width pair BOTDA for high spatial resolution sensing. Opt. Express 16, 21616-21625 (2008).

27. Soto, M. A., Bolognini, G. \& Di Pasquale, F. Long-range simplex-coded BOTDA sensor over $120 \mathrm{~km}$ distance employing optical preamplification. Opt. Lett. 36, 232-234 (2011).

28. Dong, Y. K. et al. High-spatial-resolution fast BOTDA for dynamic strain measurement based on differential double-pulse and second-order sideband of modulation. IEEE Photonics J. 5, 2600407-2600407 (2013).

29. Urricelqui, J., Sagues, M. \& Loayssa, A. Synthesis of Brillouin frequency shift profiles to compensate non-local effects and Brillouin induced noise in BOTDA sensors. Opt. Express 22, 18195-18202 (2014).

30. Tu, X. B., Luo, H., Sun, Q., Hu, X. Y. \& Meng, Z. Performance analysis of slopeassisted dynamic BOTDA based on Brillouin gain or phase-shift in optical fibers. J. Opt. 17, 105503 (2015).

31. Zhou, D. W. et al. Slope-assisted BOTDA based on vector SBS and frequencyagile technique for wide-strain-range dynamic measurements. Opt. Express $\mathbf{2 5}$, 1889-1902 (2017)

32. Soto, M. A., Ramírez, J. A. \& Thévenaz, L. Intensifying the response of distributed optical fibre sensors using 2D and $3 \mathrm{D}$ image restoration. Nat. Commun. 7, 10870 (2016).

33. Song, K. Y. \& Hotate, K. Distributed fiber strain sensor with 1-kHz sampling rate based on Brillouin optical correlation domain analysis. IEEE Photonics Technol. Lett. 19, 1928-1930 (2007). 
34. Zhang, C. Y., Kishi, M. \& Hotate, K. 5000 points/s high-speed random accessibility for dynamic strain measurement at arbitrary multiple points along a fiber by Brillouin optical correlation domain analysis. Appl. Phys. Express 8, 042501 (2015).

35. Sun, Q., Tu, X. B., Sun, S. L. \& Meng, Z. Long-range BOTDA sensor over 50 km distance employing pre-pumped Simplex coding. J. Opt. 18, 055501 (2016).

36. Dong, Y. K., Chen, L. \& Bao, X. Y. Time-division multiplexing-based BOTDA over $100 \mathrm{~km}$ sensing length. Opt. Lett. 36, 277-279 (2011).

37. Gyger, F., Rochat, E., Chin, S., Niklès, M. \& Thévenaz, L. Extending the sensing range of Brillouin optical time-domain analysis up to $325 \mathrm{~km}$ combining four optical repeaters. Proceedings of the 23rd International Conference on Optical Fibre Sensors p91576Q (SPIE, Santander, Spain, 2014).

38. Dong, Y. K., Chen, L. \& Bao, X. Y. Extending the sensing range of Brillouin optical time-domain analysis combining frequency-division multiplexing and in-line EDFAs. J. Light Technol. 30, 1161-1167 (2012).

39. Peled, Y., Motil, A., Yaron, L. \& Tur, M. Slope-assisted fast distributed sensing in optical fibers with arbitrany Brillouin profile. Opt. Express 19, 19845-19854 (2011).

40. Motil, A., Danon, O., Peled, Y. \& Tur, M. Pump-power-independent double slope-assisted distributed and fast Brillouin fiber-optic sensor. IEEE Photonics Technol. Lett. 26, 797-800 (2014).

41. Minardo, A., Coscetta, A., Bernini, R. \& Zeni, L. Heterodyne slope-assisted Brillouin optical time-domain analysis for dynamic strain measurements. J. Opt. 18, 025606 (2016)

42. Bernini, R., Minardo, A. \& Zeni, L. Dynamic strain measurement in optical fibers by stimulated Brillouin scattering. Opt. Lett. 34, 2613-2615 (2009).

43. Voskoboinik, A., Yilmaz, O. F., Willner, A. W. \& Tur, M. Sweep-free distributed Brillouin time-domain analyzer (SF-BOTDA). Opt. Express 19, B842-B847 (2011).

44. Voskoboinik, A. et al. Frequency-domain analysis of dynamically applied strain using sweep-free Brillouin time-domain analyzer and sloped-assisted FBG sensing. Opt. Express 20, B581-F586 (2012).

45. Feng, Z. H. et al. Performance-enhanced direct detection optical OFDM transmission with CAZAC equalization. IEEE Photonics Technol. Lett. 27, 1507-1510 (2015).

46. Zhao, C. et al. BOTDA using channel estimation with direct-detection optical OFDM technique. Opt. Express 25, 12698-12709 (2017).
47. Fang, J., Xu, P. B., Dong, Y. K. \& Shieh, W. Single-shot distributed Brillouin optical time domain analyzer. Opt. Express 25, 15188-15198 (2017).

48. Urricelqui, J., Sagues, M. \& Loayssa, A. BOTDA measurements tolerant to nonlocal effects by using a phase-modulated probe wave and RF demodulation. Opt. Express 21, 17186-17194 (2013).

49. Yang, G. Y., Fan, X. Y. \& He, Z. Y. Strain dynamic range enlargement of slopeassisted BOTDA by using Brillouin phase-gain ratio. J. Light. Technol. 35, 4451-4458 (2017).

50. Peled, Y., Motil, A. \& Tur, M. Fast Brillouin optical time domain analysis for dynamic sensing. Opt. Express 20, 8584-8591 (2012).

51. Kito, C., Takahashi, H., Toge, K. \& Manabe, T. Dynamic strain measurement of 10-km fiber with frequency-swept pulsed BOTDA. J. Light Technol. 35, 1738-1743 (2017).

52. Urricelqui, J., Zornoza, A., Sagues, M. \& Loayssa, A. Dynamic BOTDA measurements based on Brillouin phase-shift and RF demodulation. Opt. Express 20, 26942-26949 (2012).

53. Ba, D. X. et al. Dynamic distributed Brillouin optical fiber sensing based on dual-modulation by combining single frequency modulation and frequencyagility modulation. IEEE Photonics J. 9, 7102908 (2017).

54. Dong, Y. K. et al. Characterization of evolution of mode coupling in a gradedindex polymer optical fiber by using Brillouin optical time-domain analysis. Opt. Express 22, 26510-26516 (2014).

55. Dong, Y. K., Zhang, H. Y., Chen, L. \& Bao, X. Y. $2 \mathrm{~cm}$ spatial-resolution and $2 \mathrm{~km}$ range Brillouin optical fiber sensor using a transient differential pulse pair. Appl. Opt. 51, 1229-1235 (2012).

56. Xu, P. B. et al. Bend-insensitive distributed sensing in singlemode-multimodesinglemode optical fiber structure by using Brillouin optical time-domain analysis. Opt. Express 23, 22714-22722 (2015).

57. Boyd, R. W. Nonlinear Optics 429-471 (Academic Press, Burlington, 2008).

58. Farahani, M. A., Castillo-Guerra, E. \& Colpitts, B. G. Accurate estimation of Brillouin frequency shift in Brillouin optical time domain analysis sensors using cross correlation. Opt. Lett. 36, 4275-4277 (2011).

59. Wang, F., Zhan, W. W., Zhang, X. P. \& Lu, Y. G. Improvement of spatial resolution for BOTDR by iterative subdivision method. J. Light Technol. 31, 3663-3667 (2013). 